Supplementary Material

\title{
Yeast Heme Oxygenases: Functional Expression and Characterization of Hmx1 from Saccharomyces cerevisiae and CaHmx1 from Candida albicans $\dagger$
}

Donghak Kim ${ }^{\ddagger}$, Erik T. Yukl§, Pierre Moënne-Loccoz $\S$, and Paul R. Ortiz de Montellano ${ }^{\ddagger *}$

From the ${ }^{\ddagger}$ Department of Pharmaceutical Chemistry, University of California, San Francisco, California 94143-2280, and §Department of Environmental and Biomolecular Systems, OGI School of Science and Engineering, Oregon Health Sciences University, Beaverton, Oregon 97006-8921 


\section{$\mathrm{Hmx1}$}

\section{S M W}

\section{CaHmx1}

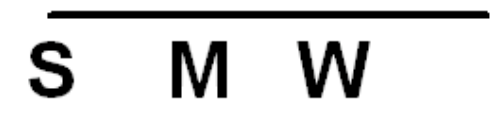

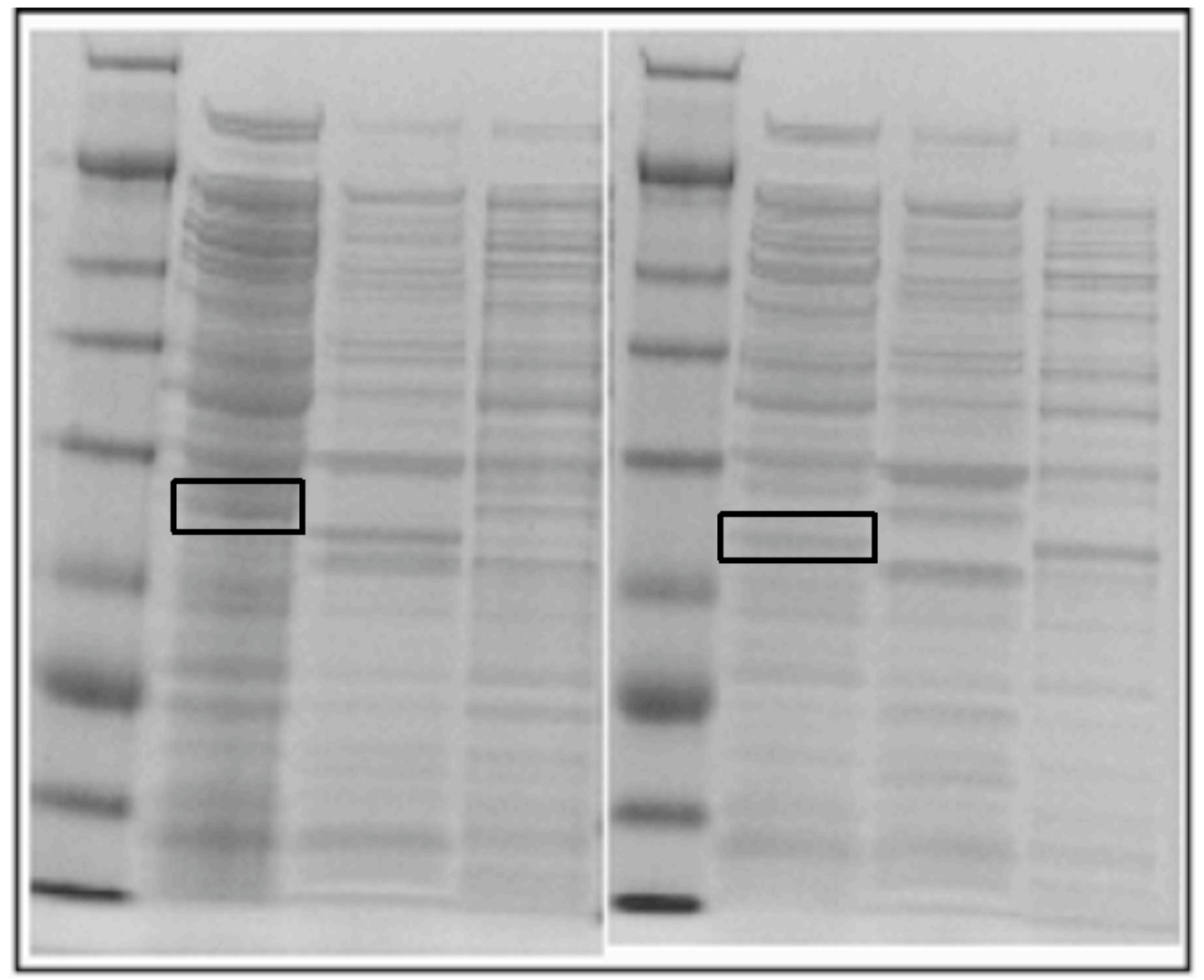

FIGURE S1. SDS-PAGE of the truncated proteins. S, soluble fraction, M, membrane fraction, and $\mathrm{W}$, whole cells. 


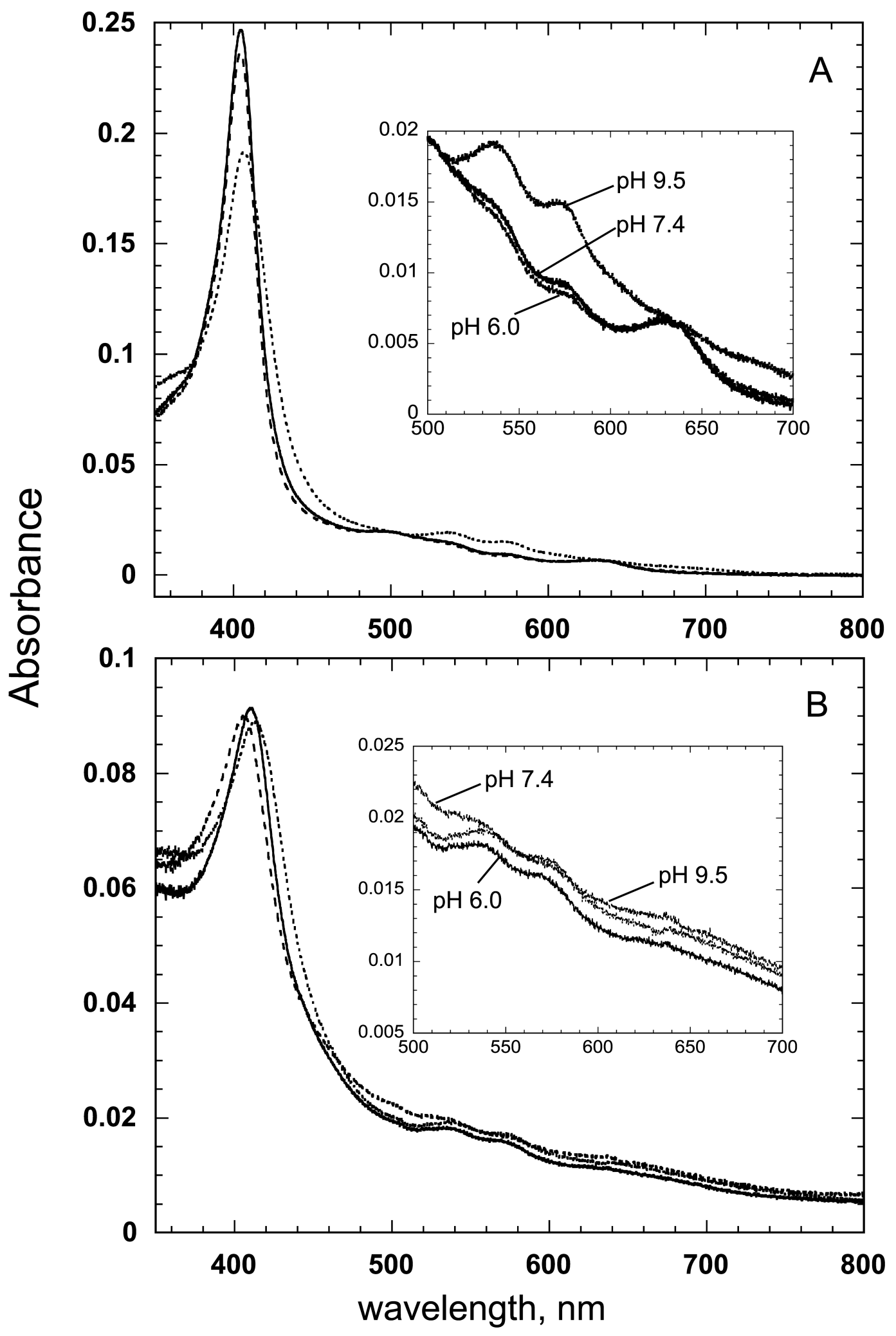

FIGURE S2. UV/visible absorption spectra of the ferric wild type Hmx1 and CaHmx1 at the varied pH conditions. pH 6.0 ( $\square \square), \mathrm{pH} 7.4$ ( $\square$ ), and pH 9.5 (----). 


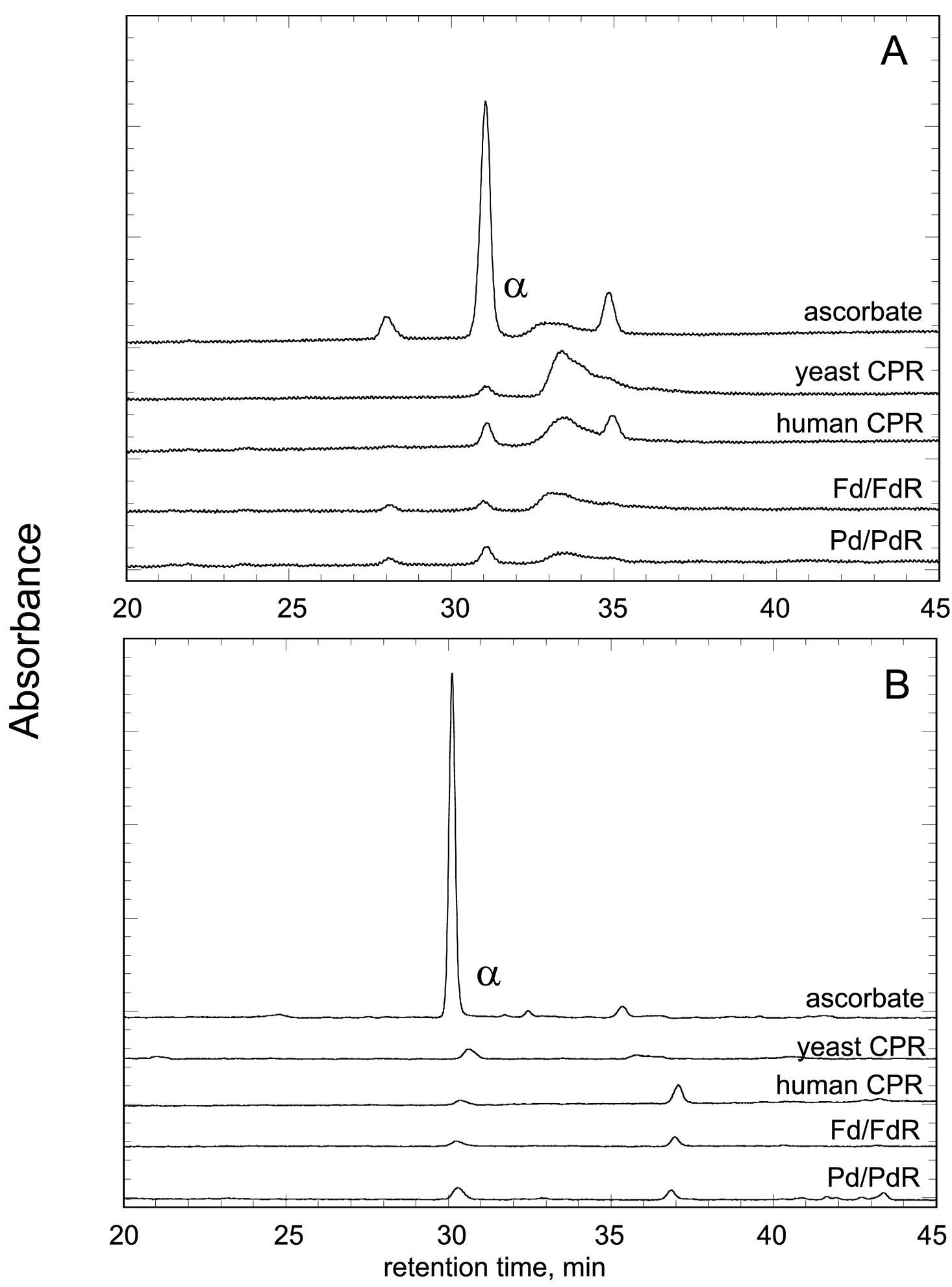

FIGURE S3. HPLC analysis of the biliverdin dimethyl esters isolated from the biliverdin formation reactions supported by several reduction partners. 


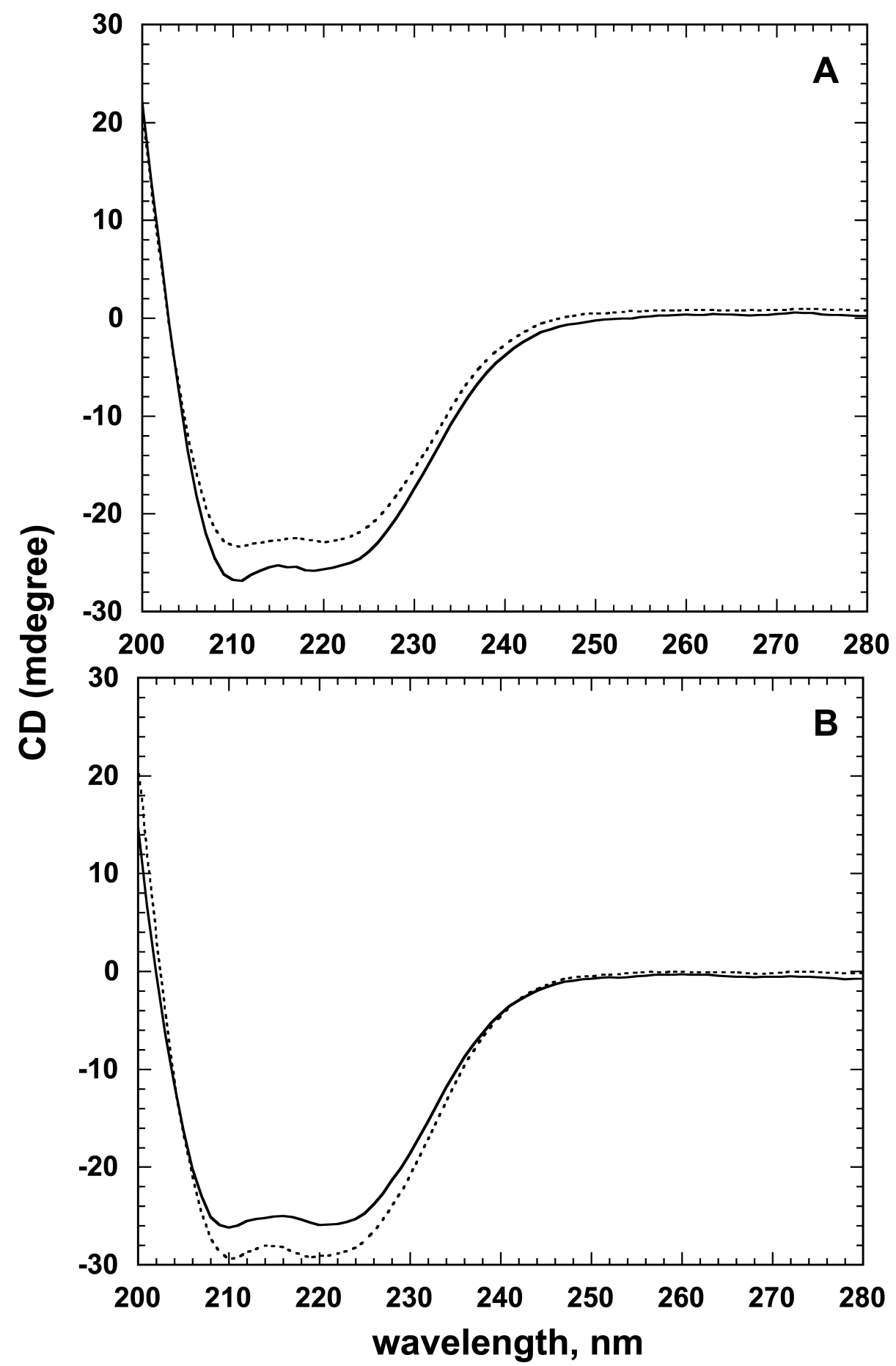

FIGURE S4. Far-UV CD spectra of wild type and mutant heme oxygenase proteins. A. Hmx1, B. CaHmx1. The wild types are indicated by solid lines while the mutants are indicated by dashed lines 


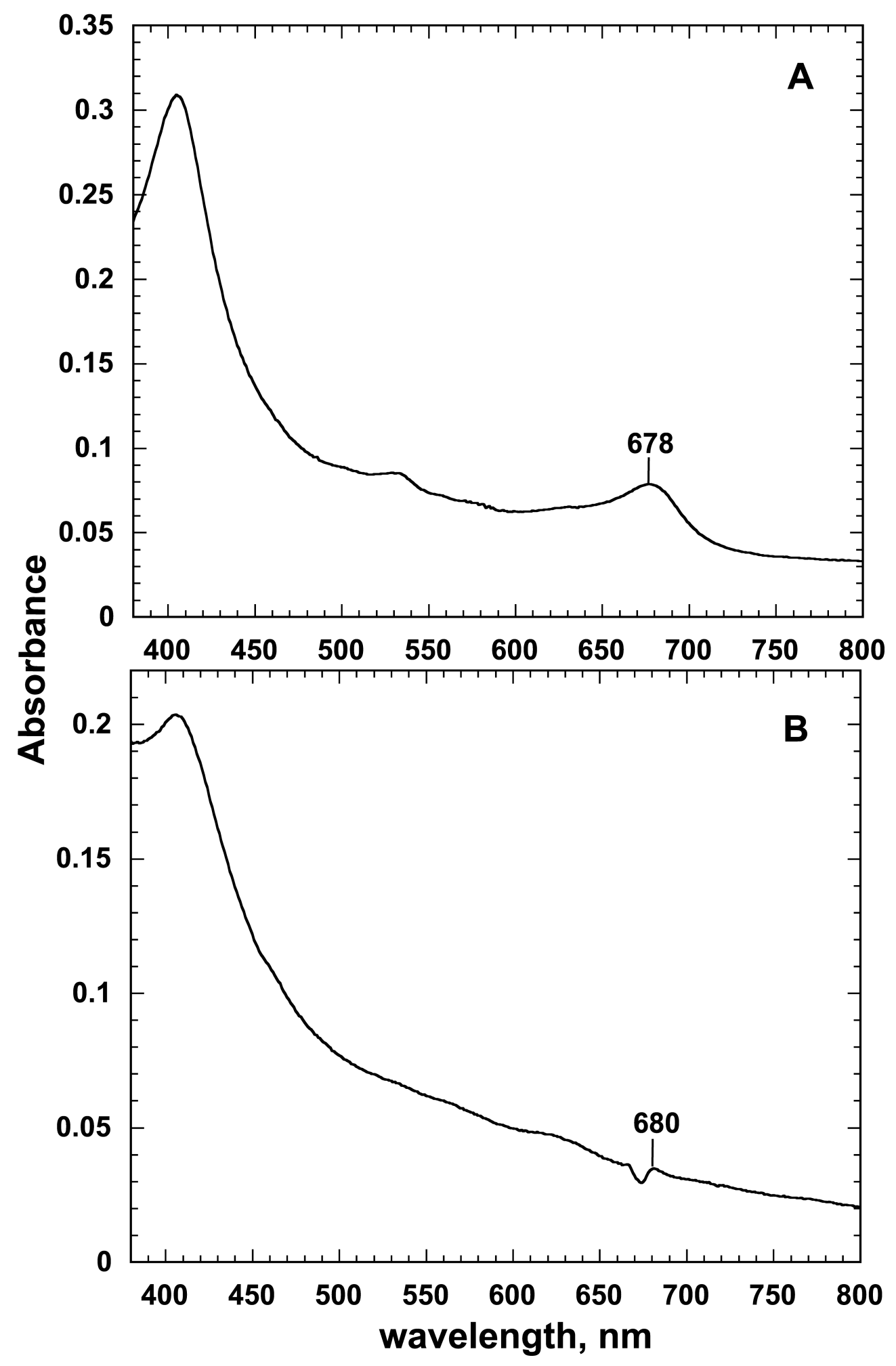

FIGURE S5. UV/visible absorption spectra of pyridine verdohemochromes. A. Hmx1, B. CaHmx1. 